\title{
XII. Resümee: Äußerer Druck und innere Rezeptoren in der Erinnerungskultur
}

Lassen sich aus den so unterschiedlichen Prozessen der „Vergangenheitsbewältigung " in Japan und (West-)Deutschland generalisierende Schlüsse ziehen für ein globales Modell von Erinnerungskultur? Oder erweist sich der mentale Graben zwischen derart entfernten Zivilisationen - trotz ihrer gemeinsamen demokratischen Grundlagen - hierfür letztlich doch als zu tief? Um den Blick für die möglichen Antworten auf diese Frage zu schärfen, sollen die wesentlichen Ergebnisse der Untersuchung zunächst noch einmal zusammenfassend gedeutet werden.

Der japanische Umgang mit der Erblast der ultranationalistischen und militaristischen Vergangenheit war über mindestens drei Dekaden hinweg durch ein schwaches Unrechtsbewußtsein gekennzeichnet, eine lange „Phase der Ignoranz"1, während der „ausgeprägtes Opferbewußtsein“2 auf der Grundlage der Hiroshima-Erfahrung dominierte und sich der Diskurs über historische Schuld vor allem gegen die Verantwortlichen jenes Regimes richtete, das die Katastrophe der militärischen Niederlage, weniger den Krieg als solchen, zu verantworten hatte. In klarem Kontrast dazu stand die entschiedene Distanzierung vom Nationalsozialismus und das frühe öffentliche Bekenntnis von Kollektivscham in Westdeutschland - auch wenn es anfänglich noch recht diffus und kaum konkret auf den Holocaust selbst bezogen war und auch wenn vor allem Millionen mehr oder weniger stark in das NS-Unrechtssystem verstrickter Deutscher der Frage nach individueller Verantwortung oft auswichen. Dabei gewann die Erinnerung an positive Erfahrungen im Dritten Reich - vom Abbau der Arbeitslosigkeit bis zum $\mathrm{KdF}$-Tourismus - einen im historisch-objektiven Blick auf die Gesamtepoche ganz und gar unangemessenen Stellenwert, und es mangelte lange auch an der Fähigkeit, sich mit den unbeschreiblichen Schrecken des Holocaust über dämonisierende Formeln der Bestürzung hinaus ganz konkret und im Blick auf antisemitische Traditionen in der deutschen Gesellschaft auseinanderzusetzen. Dennoch ging die klare Distanzierung vom überwundenen Nationalsozialismus - ebenso wie die scharfe Abgrenzung von dem anhal-

1 Fuhrt, Erzwungene Reue, S. 188. Vgl. auch das Urteil von Charles S. Maier, wonach die Japaner, anders als die Deutschen, von der Leugnung ihres Verhaltens während des Weltkriegs zu Selbstgerechtigkeit und Konformität übergegangen seien. Maier, Foreword, S. VII ff.

2 Fuhrt, Erzwungene Reue, S. 189. 
tend bedrohlichen Kommunismus - in einen neuen, von der SPD bis zu DP und BHE reichenden antitotalitären Grundkonsens der Gesellschaft ein ${ }^{3}$. Die im Vergleich zum japanischen Fall von Anfang an viel tiefer greifende Form der westdeutschen „Vergangenheitsbewältigung“ war durch eine Reihe zusammenwirkender Faktoren bedingt.

Zunächst sind die Spezifika der zu bewältigenden Verbrechenskomplexe zu sehen. Das nationalsozialistische Gewaltverbrechen „Holocaust" hatte wegen der Art seiner Planung und seiner - teilweise industriellen - Durchführung viel stärker die Dimension einer Apokalypse der Moderne als dies für die japanischen „Kriegsverbrechen“ gilt, die trotz ihrer unermeßlichen Grausamkeit und ihres partiell ebenfalls genozidalen Charakters letztlich konventioneller erschienen. Die davon ausgehenden erinnerungskulturellen Effekte wurden noch dadurch verstärkt, daß die japanischen zivilen Opfer infolge der Atombombenexplosion von Hiroshima und Nagasaki eine ganz andere Wirkung auf die nationale wie auf die Weltöffentlichkeit entfalteten als die deutschen zivilen Opfer des systematischen alliierten Bombenkriegs und des vom Umfang her ,größte[n] Vertreibungsgeschehen[s] der Weltgeschichte" 4 : Dresden und Nemmersdorf waren nicht Hiroshima ${ }^{5}$, und Nanking war nicht Auschwitz.

Auch vor diesem Hintergrund fielen die (personellen) Entnazifizierungsmaßnahmen der Besatzungsmächte in Deutschland - unter dem zumindest kurzfristig wirksamen Schock der Befreiung der Konzentrationslager durchgreifender aus als die Entmilitarisierung in Japan; zumal der Oberkommandierende General MacArthur erfolgreich verhinderte, daß die dem japanischen, "gelben" Hauptkriegsgegner gegenüber besonders feindlichen Empfindungen der Bürger und Politiker im fernen Amerika besatzungspolitisch durchschlugen. Vor allem sorgte der eigenwillige US-Prokonsul maßgeblich dafür, den Tennô Hirohito nicht vor das Tokioter Militärtribunal für den Fernen Osten zu bringen, sondern ihn auf dem Thron zu halten, um mit seiner Hilfe die besonders schwierige Aufgabe der Demokratisierung eines „orientalischen Volkes“ besser lösen zu können.

Bald zeitigten auch die Konstellationen des Kalten Krieges in Europa und im Fernen Osten erinnerungskulturell ganz gegensätzliche Folgen. Im Kampf um die Bewahrung der Freiheit gegen die kommunistische Bedro-

3 Der von Winkler in diesem Zusammenhang gebrauchte Begriff des „liberalen Grundkonsenses“ (Winkler, Der lange Weg nach Westen, S. 439) scheint mißverständlich, da dieser Konsens in seinen wesentlichen Elementen nicht nur von liberalen und linken, sondern auch von konservativen Demokraten mitgetragen wurde.

4 Pfeil, Zentrum, S. 124. Im bedauerlichen Streit um ein europäisch ausgerichtetes „Zentrum gegen Vertreibungen " in Berlin sind die Folgen dicses Phänomens noch heute zu besichtigen; vgl. Kittel, Preußens Osten in der Zeitgeschichte, vor allem S. 446 f., 462 . Vgl. auch Hirsch, Flucht und Vertreibung, vor allem S. 14, 25.

5 Vgl. dagegen den reißerischen Untertitel des Buches von McKee, Dresden 1945, der von einem "deutschen Hiroshima“ spricht. 
hung entstand in Westeuropa unter Einschluß der Bundesrepublik eine wirtschafts- und verteidigungspolitische Staatengemeinschaft, die dem westdeutschen Staat Rücksicht auf internationale Empfindlichkeiten, nicht zuletzt in bezug auf die NS-Vergangenheit, ins Stammbuch schrieb, während Japan nach 1945 seinen nationalen Sonderweg - wenn auch jetzt unter ausschließlicher Anlehnung an die USA - als einziges großes Bollwerk gegen den Kommunismus in Ostasien weitergehen konnte oder mußte. Zudem blieb die staatliche Einheit Japans erhalten, während die Teilung Deutschlands bzw. das Festhalten am schwierigen Ziel der Wiedervereinigung und den damit verknüpften Rechtspositionen die Bundesrepublik gegenüber äußerem Druck seitens der alliierten Mächte besonders anfällig machte. Japan sah sich dagegen viel geringerem Außendruck ausgesetzt.

Das im Krieg am stärksten geschädigte chinesische Volk war durch den in der Gründung Taiwans verstetigten Gegensatz zwischen kommunistischer und nationalistischer Regierung bis 1972 daran gehindert, erfolgreich erinnerungskulturelle Forderungen geltend zu machen - gerade auch im zentralen Bereich der „Wiedergutmachung“ für die Opfer von Verbrechen gegen die Menschlichkeit. Versuche Südkoreas, der zweiten großen Opfernation, Japan zur Anerkennung seiner historischen Verantwortung zu bewegen, scheiterten ebenfalls. Weder unterstützten die USA als wichtigster Bündnispartner beider Staaten die Forderungen des weit kleineren Südkorea hinreichend, noch gab es auf japanischer Seite wirkungsvolle Akteure, die den von Seoul ausgeübten Außendruck gleichsam von innen verstärkt hätten - so wie das etwa bei der Frage der „Wiedergutmachung“ in der Bundesrepublik seitens engagierter Kräfte aus verschiedenen politischen Lagern mit den Ansprüchen der Jewish Claims Conference geschah.

War die Bundesrepublik schon objektiv höherem Außendruck durch Vertreter der größten Opfergruppe des NS-Rassenwahns ausgesetzt - nicht nur, aber auch infolge der Wachsamkeit amerikanisch-jüdischer Organisationen -, so wurde deren Einfluß auf Konzeption und Durchführung der US-Deutschlandpolitik in den 1950er Jahren in der subjektiven Wahrnehmung bundesdeutscher Zeitgenossen noch dadurch vermehrt, daß sie ihn unter der Nachwirkung völkisch-antisemitischer Propaganda beträchtlich überschätzten. Denn tatsächlich vermochten sich die Anliegen der jüdischen Minderheit in der pluralistischen Demokratie der Vereinigten Staaten, deren Interessen vor allem auf das Bestehen des Kalten Krieges gerichtet waren, vor dem Einsetzen der Ost-West-Entspannung und dem Eskalieren des Nahost-Konflikts 1967/73 lange nicht stärker Geltung zu verschaffen. Andererseits ist unübersehbar, daß eine dem - zu relativierenden - Einfluß der jüdisch-amerikanischen Verbände irgendwie ähnliche, auch nur potentielle Druckkulisse von der winzigen chinesischen oder gar koreanischen Minderheit in den USA nicht aufgebaut werden und folglich auch keine Wirkungen auf Japan entfalten konnte. 
Die genannten außenpolitischen Bedingungsfaktoren waren in der Bundesrepublik für das erinnerungskulturelle Engagement der von 1949 bis 1969 regierungsführenden Unionsparteien kaum weniger wichtig als die offensichtliche innere Wandlung des deutschen Konservativismus aufgrund der Erfahrung des NS-Regimes. Denn parteipolitisch setzte sich dieser nur gebrochen fort und verschmolz mit christlich-sozialen und liberalen Elementen in CDU und CSU zu einer bürgerlichen Sammlungspartei der Mitte, deren erste Garde im Dritten Reich oft genug persönlich verfolgt worden war und die nationale Zukunft fürderhin in europäischer Integration und Westbindung suchte. Ähnliche Erfahrungen hatten die japanischen Konservativen in einem Land ohne Widerstand während des Ultranationalismus so gut wie überhaupt nicht gemacht und als um so stärker erwiesen sich hier die geistigen Kontinuitäten. Was für den regierenden Konservativismus gilt, zeigt sich auch beim Vergleich der (linken) Opposition in Japan und Westdeutschland. Denn zur einmütigen Distanzierung der politischen Klasse vom Nationalsozialismus trug die Haltung der Sozialdemokratie, in deren Reihen besonders viele Ex-Verfolgte für eine gründliche „Vergangenheitsbewältigung" stritten, erheblich bei, während die japanische Linke nicht nur politisch insgesamt schwächer, sondern auch erinnerungskulturell passiver war, sei es, weil sie aus den einschlägigen Themata wahltaktisch wenig Kapital schlagen zu können meinte, sei es, weil sie einen antiamerikanisch-nationalistisch grundierten Pazifismus ausbildete, für den die - eigenen - Opfer von Hiroshima im Mittelpunkt standen, nicht aber irgendwelche Verbrechen, die im Ausland gegen Fremde begangen worden waren.

Die Reichweite linksoppositioneller Bewältigungskritik war von vornherein auch dadurch begrenzt, daß die Medien in Japan nach 1945 qua Besatzungspolitik viel weniger stark neuausgerichtet worden waren als in der Bundesrepublik. Zudem hatte die zeitgeschichtliche Forschung - nicht nur aus archivalischen Gründen - unter wesentlich schwierigeren Bedingungen beginnen müssen. Darüber hinaus erwies sich die Dominanz der Marxisten innerhalb der japanischen Historikerschaft nach 1945 in doppelter Hinsicht als erinnerungskulturell kontraproduktiv. Zum einen wegen der Binnenfixierung auf sozioökonomische Fehlentwicklungen des japanischen Sonderwegs, die allenfalls noch die gesellschaftlichen Bedingungen der Kolonialpolitik, nicht aber die genozidale Kriegsführung in China zu einem wichtigen Untersuchungsgegenstand werden ließ; zum anderen wegen der allzu weiten Entfernung der nationalkritischen marxistischen Historiker vom zutiefst konservativen Geleitzug der japanischen Gesellschaft. Ein Umstand, der vor allem im Vergleich zur Bundesrepublik ins Auge fällt, wo die dominierenden konservativen Geschichtswissenschaftler auch deshalb viel mehr Einfluß hatten, weil sie im gesellschaftlichen „Mainstream“ blieben, die Vorstellung von einem Sonderweg ins Dritte Reich zurückwiesen, die Diskontinuität zwischen der nationalen Außenpolitik Bismarcks oder Strese- 
manns und dem nationalsozialistischen Lebensraumwahn Hitlers stark betonten, sich aber gleichzeitig auch scharf vom System des Dritten Reiches und seinen Verbrechen distanzierten und dadurch zu dessen Stigmatisierung beitrugen. Dies wurde auch in den Schulbüchern der Bundesrepublik im Laufe der 1950er Jahre immer spürbarer, während in Japan nach dem Abzug der USA 1952 in einer gegenläufigen Entwicklung ein erinnerungskultureller Rückfall erfolgte und die Konfrontation mit den Schatten der Vergangenheit in den Bildungsanstalten immer weniger Platz fand.

Nachdem Schule und Medien, Regierung und Parlament in Japan kaum zeithistorisches Problembewußtsein vermittelten und „Vergangenheitsbewältigung " in der politischen Sozialisation der jungen Generation während der 1950er und 1960er Jahre wenig Bedeutung hatte, bildete nicht einmal auf dem extrem linken Flügel der japanischen „68er“, bei der nationalen Studentenorganisation des Zengakuren, der Rekurs auf den japanischen „Faschismus" und seine Verbrechen ein inhaltlich tragendes oder auch nur taktisch vorgeschobenes Motiv des Protests. Viel wichtiger für die Studentenbewegung, die schon 1960 im Kampf gegen eine unzureichende Revision des „ungleichen“ amerikanisch-japanischen Sicherheitsvertrages ihren ersten Höhepunkt erlebte, waren Motive rein pazifistischer Art.

Der Zusammenhang zwischen der geringen gesamtgesellschaftlichen Aufmerksamkeit für die ultranationalistische Vergangenheit und der mangelnden erinnerungskulturellen Protestmotivation der Studentenbewegung in Japan hat seine Logik. Überträgt man sie auf die Bundesrepublik, so nährt dies Zweifel an dem archaischen Deutungsmuster, der Protest der westdeutschen „68er“ sei vor allem die Antwort auf eine reale Verdrängungspraxis in der Zeit vorher gewesen. Denn die Hoffnung, die Bundesrepublik durch den Nachweis von Kontinuitäten zur „faschistischen“ Vergangenheit erschüttern zu können, vermochte sich offensichtlich kaum aus der Wahrnehmung zu speisen, die politisch interessierte Öffentlichkeit stünde der verbrecherischen NS-Geschichte vollständig blind und fühllos gegenüber, sondern ganz im Gegenteil nur aus dem hohen Grad an Aufmerksamkeit, den der Komplex, wenn auch nicht permanent, aber doch in immer neuen Schüben seit Gründung der Bundesrepublik - vor allem dann infolge der antisemitischen "Schmierwelle“ um 1959/60 und der großen NS-Prozesse - erzeugt hatte.

Die öffentliche Auseinandersetzung mit der Vergangenheit war tatsächlich oft mit individueller Verdrängung auf der privaten Ebene einher gegangen, d.h. mit dem Beschweigen bestimmter Kapitel der eigenen Biographie oder der des Nächsten. Ein „weitverbreitetes Verstummen um den Holocaust" kennzeichnete im übrigen während des ersten Jahrzehntes nach der Staatsgründung 1948 auch Israel, das „Land der Opfer“, wobei die viel- 
schichtigen Gründe hierfür anders lagen als im „Land der Täter"6. Doch kann für die Bundesrepublik von „privater Diskretion“ gegenüber der NSZeit keineswegs auf die Gesamtentwicklung geschlossen werden, zumal „eine öffentliche Wirkung von den publizierenden, nicht den privatisierenden Kreise[n] ausgeht."7. So problematisch die „Vergangenheitsbewältigung“ in der Ära Adenauer vor allem hinsichtlich der Karrieren allzu stark Belasteter aus dem Kreis der ehemaligen „NS-Eliten“ in Justiz und Polizei partiell auch war ${ }^{8}$, so wenig gab es zu einer Politik der Integration aufs Ganze gesehen doch eine Alternative. Ob Hans-Ulrich Wehlers davon abweichende Äußerung, die riesige Zahl der NS-Mitläufer hätte „an den Rand der Gesellschaft " gedrängt werden sollen" ${ }^{9}$, mit dem Ziel vereinbar gewesen wäre, ein demokratisches Gemeinwesen aufzubauen, ist zweifelhaft ${ }^{10}$. Schließlich legte wohl gerade die Akzeptanz des "geduckten Opportunismus" der einstigen Führungsgruppe des Dritten Reiches durch die neue Demokratie bei nicht wenigen die "Grundlage zu einem tatsächlichen Einstellungswandel“11.

Es ist bemerkenswert, daß der japanischen Demokratie selbst eine viel weitergehende Integration der Ehemaligen nicht existentiell geschadet hat, auch wenn ihre anhaltend paternalistisch-oligarchisch-bürokratischen Strukturen wohl nur im Ergebnis der eher oberflächlichen personellen und ideellen „Vergangenheitsbewältigung“ zu erklären sind. Immerhin aber haben die Erfahrung von Hiroshima und der Prozeß der Entmilitarisierung im Lande der Samurai pazifistische Grundideen feste Wurzeln schlagen lassen und insofern eine tiefgreifende Veränderung der politischen Kultur bewirkt. Das Urteil, Japans Umgang mit der Vergangenheit sei einfach „infantil“12, kann jedenfalls nicht überzeugen, weil es zu stark aus der Perspektive abendländischer Erinnerungspolitik argumentiert.

Der wesentliche Grundtatbestand, daß in Japan kein „Holocaust“ aufzuarbeiten war, sondern vor allem eine konventioneller wirkende „Dreistrahlen“-Kriegführung gegen China, und daß Hiroshima eine ganz andere Ausprägung des Bewußtseins bewirkte, selbst Opfer gewesen zu sein, als dies in Westdeutschland der Fall war, vermag die Unterschiede der „Vergangenheitsbewältigung“ zwischen beiden Ländern nicht ganz zu erklären. Man kann selbst zweifeln, ob der ebenfalls stark ins Gewicht fallende Faktor des divergierenden Außendrucks letztlich entscheidend war. Denn selbst in der

6 Vgl. Zuckermann, Zweierlei Holocaust, v.a. S. $177 \mathrm{f}$.

7 Fröhlich/Kohlstruck (Hg.), Engagierte Demokraten, S. 16.

${ }^{8}$ Am Beispiel der Resozialisierung von NS-Kriminalisten, deren defensive „Traditionslügen nur als dreist und moralisch unerträglich erscheinen" konnten, hat dies jetzt Patrick Wagner untersucht. Wagner, Die Resozialisierung der NS-Kriminalisten, S. 213.

9 Wehler, in: Broszat u. a., Deutschlands Weg in die Diktatur, S. 359.

10 So auch Kielmansegg, Lange Schatten, S. $22 \mathrm{f}$.

11 Herbert, NS-Eliten in der Bundesrepublik, $114 \mathrm{f}$.

12 Buruma, Erbschaft der Schuld, S. $365 \mathrm{f}$. 
Phase des wachsenden gaiatsu ${ }^{13}$ seit den 1980er Jahren scheint sich zumindest im breiten konservativen Meinungsspektrum Japans weithin keine innere Wandlung zu einem offeneren Umgang mit dem Ultranationalismus vollzogen zu haben. Daß Außendruck vielmehr auch innerer Rezeptoren in den betroffenen Ländern selbst bedarf, um voll wirksam werden zu können, zeigt die westdeutsche Entwicklung paradigmatisch. Ohne Zweifel wäre hier im Falle einer anderen internationalen Konstellation „vieles liegen geblieben"14. Doch es gab nicht nur zahlreiche Felder der Erinnerungskultur, wo der Hinweis auf das hellhörige (westliche) Ausland zur aktivitätsbegründenden Standardformel gehörte, sondern andererseits auch eine Reihe von Themen, die überwiegend ohne Schielen nach draußen von den Bundesdeutschen selbst angepackt wurden. Wie entscheidend auch der inländische, nationale Wille zur „Aufarbeitung“ der jüngsten Vergangenheit war, zeigt sich am nachdrücklichsten bei der Verfolgung von Gewaltverbrechen, die in Westdeutschland - trotz mancher Unterlassungen und Versäumnisse - im Ganzen jahrzehntelang und mit außerordentlicher Wirkung auf das zeithistorische Bewußtsein geleistet wurde. Die Gründung der Ludwigsburger Zentralstelle zur Aufklärung von NS-Verbrechen 1958, mitten in der Ära Adenauer, erfolgte im wesentlichen nicht etwa auf äußeren Druck hin ${ }^{15}$, sondern auf Initiative besorgter Staatsanwälte und Justizpolitiker im eigenen Lande; und auch in der vorhergehenden Phase, in den am stärksten von Wiederaufbau und Westintegration, Wiederbewaffnung und „Gnadenfieber" geprägten frühen 1950er Jahren kam die Strafverfolgung nie ganz zum Erliegen, waren der sogenannten „Vergangenheitspolitik“16 stets Grenzen gesteckt. Der Unterschied zu Japan könnte gerade im Bereich der strafrechtlichen Verfolgung kaum größer sein.

Wenn also auch dem nationalen Willen zur „Vergangenheitsbewältigung“ selbst und den zur Verstärkung von Außendruck notwendigen inneren Rezeptoren personeller wie institutioneller Art ein so hohes erinnerungskulturelles Gewicht zukommt, wird man abschließend fragen müssen, weshalb es damit in Japan und Deutschland so unterschiedlich bestellt war. Die Spezifika der Verbrechenskomplexe, die Folgen von Auschwitz, Nanking - und Hiroshima - , so scheint es, verstärkten nur noch die ohnehin sehr gegensätzlichen Dispositionen beider Gesellschaften zur „Vergangenheitsbewältigung“. Die Erinnerungskulturen in Japan und Deutschland entwickelten sich dabei gleichsam in „Gefängnissen langer Dauer“ (Fernand Braudel): Sie

13 Jap. für: Außendruck.

14 Woller, Der Rohstoff des kollektiven Gedächtnisses, S. 75.

15 Die von Greve, Der justitielle und rechtspolitische Umgang, S. 52, angeführte Stellungnahme des Auswärtigen Amtes vom 20. Oktober 1958, das die Errichtung der zentralen Ermittlungsstelle ebenfalls begrüßte, greift als Gegenbeweis zu kurz.

16 Verstanden als „Bewältigung der frühen NS-Bewältigung“ (Frei, Vergangenheitspolitik, S. 13) während der Gründerjahre der Bundesrepublik. 
wurden von Menschen gepflegt bzw. nicht gepflegt, deren Mentalitäten durch eine Jahrhunderte alte shintôistische bzw. christliche Tradition wesentlich mit geprägt waren. Tief sind die mentalen Unterschiede zwischen einer christlichen, vor allem auch protestantischen, von der Frage nach Schuld und Sühne beeinflußten, nach außen offenen deutschen Erinnerungslandschaft und einer japanischen, die von verschiedenen Elementen des Shintôismus, Buddhismus und Konfuzianismus geformt ist und wegen ihrer religiösen Sinngebung der Vergangenheit „den nationalen Rahmen nicht überschreitet"17; das heißt, sie heroisiert die eigenen Kriegsgefallenen, weil diese mit ihrem heldenhaften Opfer erst die erfolgreiche Entwicklung der Nachkriegsgesellschaft ermöglicht hätten, ja mehr noch, „nun als Gottheit für das Wohlergehen der Hinterbliebenen sorgten"18, und ist aufgrund dieser Identifikation wohl außerstande, die Ahnen „auch in der Kategorie der Täter zu betrachten" 19 .

$\mathrm{Zu}$ bedenken ist schließlich, daß Werte, und dies gilt auch für den Wert der Erinnerung, stets an Kulturen gebunden sind. Maßstäbe westlicher Nationen lassen sich an Japan nicht eins zu eins anlegen. Die von anderen Religionen hervorgebrachten Wertesysteme sind jedenfalls nicht weniger legitim als das abendländische, auch wenn über ihre Gültigkeit gewiß zu streiten wäre ${ }^{20}$. Der christlich geprägte Westen glaubt - anders als noch in der frühen Neuzeit - nach der Erfahrung der Weltkriege und unter dem Einfluß Freudscher Psychoanalyse besonders fest an den Wert irdischer Gerechtigkeit, an „Katharsis" und Läuterung und an die Notwendigkeit von Trauerarbeit, die einen Schutz vor Wiederholung des schrecklichen Vergangenen schaffe. Asiatischen Zivilisationen ist dieses Denken fremd. Sie fragen vielmehr „beharrlich nach dem Nutzen der irdischen Gerechtigkeit für die soziale Harmonie und die Wohlfahrt des Gemeinwesens. Sie schauen entschlossen nach vorn“ und - sie lassen „die Vergangenheit ruhen“21.

Wenn aber gesagt worden ist, Japan verlange christlich geprägten Völkern eine „kopernikanische Wende ihres moralischen Denkens ab" 22 , so kann das nur heißen, die eigenen Maßstäbe nicht vorschnell absolut zu setzen und zu universalisieren, es kann aber nicht meinen, nun etwa das japanische „Modell“ der Bundesrepublik ex post anzuempfehlen und die unbestreitbaren Vorzüge christlicher Moral im Bereich der „Vergangenheitsbewältigung “ zu verkennen, die vor allem ungleich mehr Opfern der Diktatur ideelle und zumindest auch eine gewisse materielle Entschädigung eingebracht hat. Vielmehr wirft der Vergleich beider Entwicklungen die Frage auf, ob es bei der

\footnotetext{
17 Shimada, Formen der Erinnerungsarbeit, S. 40.

18 Ebd., S. 39.

19 Ebd., S. 38.

20 In diesem Sinne auch Lokowandt, Shintô, S. 91.

21 Scharlau, Der General und der Kaiser, S. 140.

22 Vahlefeld, Japan, S. 27.
} 
Bewältigung von Ultranationalismus und Nationalsozialismus in Japan und Deutschland nicht auch andere Wege gegeben hätte, die im einen Fall weniger stark von shintôistischer Schamvermeidung und Gesichtswahrung geprägt gewesen wären und es im anderen vermieden hätten, vom notwendigen protestantischen Schuldbekenntnis teilweise zu problematischen Formen von „Schuldstolz“ überzugehen. 